\title{
Fast IMRT with narrow high energy scanned photon beams
}

\author{
Björn Andreassen, ${ }^{\text {a) }}$ Sara Janek Strååt, and Rickard Holmberg \\ Department of Medical Radiation Physics, Karolinska Institutet and Stockholm University, \\ P.O. Box 260, SE-171 76 Stockholm, Sweden \\ Peder Näfstadius \\ Department of Hospital Physics, Karolinska University Hospital, SE-171 76 Stockholm, Sweden
}

Anders Brahme

Department of Medical Radiation Physics, Karolinska Institutet and Stockholm University, P.O. Box 260, SE-171 76 Stockholm, Sweden and Department of Hospital Physics, Karolinska University Hospital, SE-171 76 Stockholm, Sweden

(Received 24 September 2010; revised 1 July 2011; accepted for publication 2 July 2011; published 29 July 2011)

Purpose: Since the first publications on intensity modulated radiation therapy (IMRT) in the early 1980s almost all efforts have been focused on fairly time consuming dynamic or segmental multileaf collimation. With narrow fast scanned photon beams, the flexibility and accuracy in beam shaping increases, not least in combination with fast penumbra trimming multileaf collimators. Previously, experiments have been performed with full range targets, generating a broad bremsstrahlung beam, in combination with multileaf collimators or material compensators. In the present publication, the first measurements with fast narrow high energy (50 MV) scanned photon beams are presented indicating an interesting performance increase even though some of the hardware used were suboptimal.

Methods: Inverse therapy planning was used to calculate optimal scanning patterns to generate dose distributions with interesting properties for fast IMRT. To fully utilize the dose distributional advantages with scanned beams, it is necessary to use narrow high energy beams from a thin bremsstrahlung target and a powerful purging magnet capable of deflecting the transmitted electron beam away from the generated photons onto a dedicated electron collector. During the present measurements the scanning system, purging magnet, and electron collimator in the treatment head of the MM50 racetrack accelerator was used with 3-6 $\mathrm{mm}$ thick bremsstrahlung targets of beryllium. The dose distributions were measured with diodes in water and with EDR2 film in PMMA. Monte Carlo simulations with GEANT4 were used to study the influence of the electrons transmitted through the target on the photon pencil beam kernel.

Results: The full width at half-maximum (FWHM) of the scanned photon beam was $34 \mathrm{~mm}$ measured at isocenter, below $9.5 \mathrm{~cm}$ of water, $1 \mathrm{~m}$ from the $3 \mathrm{~mm}$ Be bremsstrahlung target. To generate a homogeneous dose distribution in a $10 \times 10 \mathrm{~cm}^{2}$ field, the authors used a spot matrix of 100 equal intensity beam spots resulting in a uniformity of collimated $80 \%-20 \%$ penumbra of $9 \mathrm{~mm}$ at a primary electron energy of $50 \mathrm{MeV}$. For the more complex cardioid shaped dose distribution, they used 270 spots, which at a pulse repetition frequency of $200 \mathrm{~Hz}$ is completed every $1.36 \mathrm{~s}$.

Conclusions: The present measurements indicate that the use of narrow scanned photon beams is a flexible and fast method to deliver advanced intensity modulated beams. Fast scanned photon IMRT should, therefore, be a very interesting modality in the delivery of biologically optimized radiation therapy with the possibility for in vivo treatment verification with PET-CT imaging.

(C) 2011 American Association of Physicists in Medicine. [DOI: 10.1118/1.3615059]

Key words: IMRT, scanned photon beams, Monte Carlo

\section{INTRODUCTION}

The introduction of physically and biologically optimized intensity modulated radiation therapy (IMRT) into clinical practice has stimulated the development of faster, high resolution multileaf collimators and more recently fast dynamic multileaf collimation (DMLC) ${ }^{1-3}$ or penumbra trimming multileaf collimation (PMLC). ${ }^{4,5}$ Using DMLC to modulate an incoming broad photon beam is a proven technique for delivering IMRT and has been the subject of improvement and development since the early 1980s. Conventional treatment units delivering 4-20 MV photon beams generally use flattening filters to create a homogeneous flat broad photon beams, which then can be shaped by segmental multileaf collimation (MLC) or DMLC. With a treatment unit based on the scanning beam technique in combination with a PMLC instead of a flattening filter and DMLC, a more flexible and faster system for IMRT is possible. A scanning system is already available with the racetrack microtron MM50, ${ }^{6}$ where the intrinsic electron beam from 5 up to 50 
$\mathrm{MeV}$ is stigmatically scanned, so that the focus of the resulting bremsstrahlung beam is a fixed point source just below the upstream side of the bremsstrahlung target as shown in Fig. 1(a). Generally, a full range bremsstrahlung target, where no primary electrons are transmitted through the target, is used and the photon produced electrons and positrons that are ejected from the downstream side of the target are removed by a strong purging magnet. Even though the treatment unit was originally designed for general bremsstrahlung scanning, ${ }^{6-9}$ the clinical practice has been to create uniform photon beams by scanning the bremsstrahlung lobe in a quasicircular pattern corresponding to the desired beam diameter. The final field shape is then fine adjusted by the setting of the MLC. By altering the scan pattern and only delivering pulses at optimal positions inside the collimated area, the field can be more accurately and rapidly modulated than with just the MLC. The leakage of secondary photons, the treatment time for small fields, and the secondary radiation from the treatment head can be reduced by not simply relying on the mechanical movement of the collimator

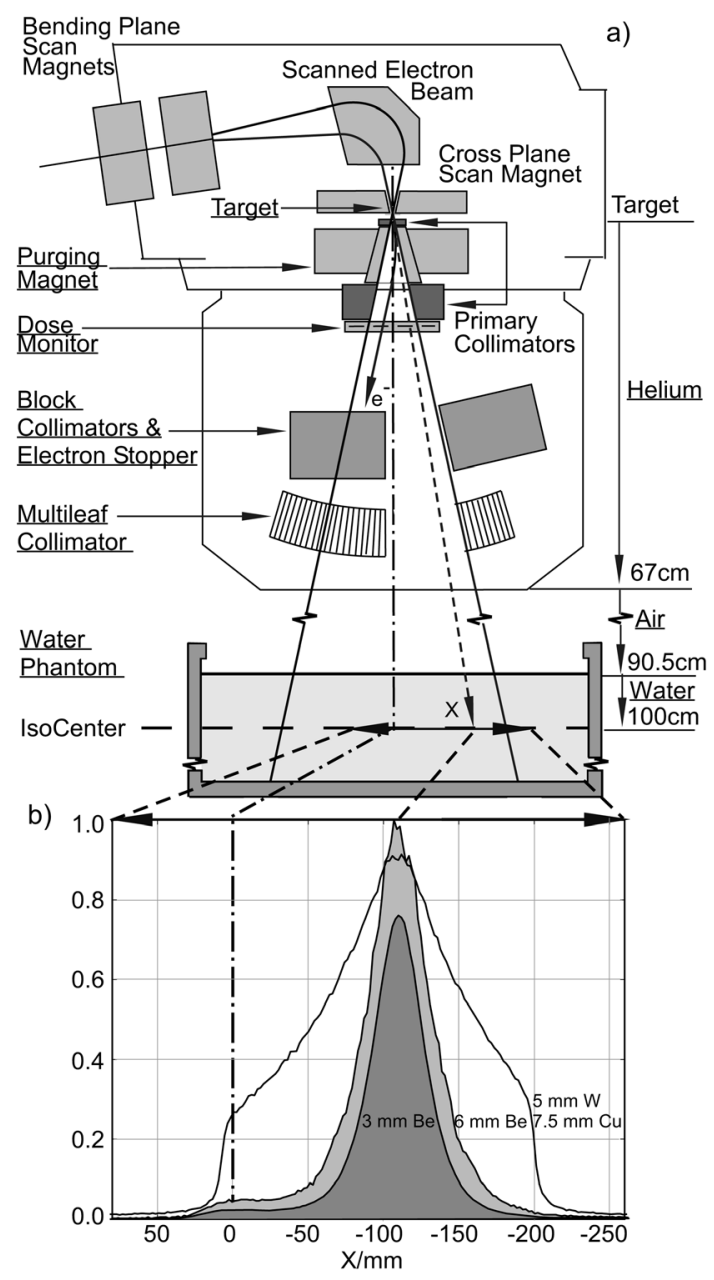

FIG. 1. (a) Schematic view of the treatment head and the used setup for the experiments. The underlined objects were accounted for in the Monte Carlo simulations. (b) Measured scanned bremsstrahlung kernels for $50 \mathrm{MeV}$ electrons incident on $3 \mathrm{~mm} \mathrm{Be}, 6 \mathrm{~mm} \mathrm{Be}$, and $5 / 7.25 \mathrm{~mm} \mathrm{~W} / \mathrm{Cu}$ at isocenter below $95 \mathrm{~mm}$ of water. The measurements are normalized to the forward photon yield of $6 \mathrm{~mm} \mathrm{Be}$ and are made in a plane $8.5 \mathrm{~cm}$ from the central axis to avoid the vacuum window contamination. leaves. When using scanned beams, the achievable pencil beam half width and the complexity of the delivered dose distributions are improved by increasing the intrinsic electron beam energy and decreasing the source to tumor distance and the thickness of the bremsstrahlung target. ${ }^{8,10} \mathrm{~A}$ conventional photon unit with an intrinsic electron energy of 6-18 MeV is thus not suitable for IMRT with scanned photon beams.

The idea of using the scanned beam to modulate the field by itself or in combination with a MLC or DMLC has been discussed previously $6,7,9,11$ and some experimental work has been performed. ${ }^{8,9,12,13}$ These experiments used either a full range target or compensators reproducing the effect of the scanned beam dose distribution. With a scanned beam using a full range target, it is possible to create, for example, simple wedge shaped dose distributions without using the MLC. ${ }^{6-8}$ To be able to produce a more complex beam shapes the photon beam should be as narrow as possible, which requires a high electron energy and a thin bremsstrahlung target. However, the forward bremsstrahlung yield first increases and then after about a target thickness of 0.3 times the electron range decreases. For the present study, 3-6 mm beryllium targets were used to produce narrow bremsstrahlung beams. A $3 \mathrm{~mm}$ beryllium target produces a very narrow photon beam with a full width at half-maximum of about $33 \mathrm{~mm}$ at a source to surface distance (SSD) of 100 $\mathrm{cm}$ (Ref. 10) and a intrinsic electron energy of $50 \mathrm{MeV}$, such a beam is well suited for scanned beam IMRT.

One of the principal problems in the medical use of high energy photon beams is the impact of the electron contamination, either produced in the air or by photon interactions with materials in the treatment head, which together will increase the entrance dose and decrease the depth of dose maximum. This has been extensively studied both for conventional treatment units and for the racetrack microtron MM50. ${ }^{6,14}$ When using a thin target, the multiple scatter of the transmitted electrons is a major concern because they have only lost a small fraction of their energy in the bremsstrahlung target. In the present study, the contribution of the transmitted electrons to the integral dose was studied by using the purging magnet to deflect the transmitted electrons onto a simple electron collector. For this purpose one of the existing block collimators in the MM50 treatment head was used, while directing or scanning the narrow photon beam in the opposite direction as shown in Fig. 1(a). Since neither the treatment head nor the purging magnet was designed for this purpose, the scattered secondary radiation from this setup is somewhat higher than desirable. However, it does give a good indication that the low levels of transmitted and elastically scattered electrons can be trapped with a dedicated electron collector without creating adverse stray radiation.

In the present study, the narrow photon beam from a 3 $\mathrm{mm}$ Be target and the scanning system of the MM50 have been used to modulate the scanned therapeutic beam both with and without using the MLC. The desired dose distribution and the measured elementary narrow photon pencil beam kernel were used as input for inverse therapy planning. The resulting optimally deliverable continuous kernel 
distribution was then discretized into a manageable number of equal strength scanning positions. In the present study, the scanning system, the deliverable dose distributions as well as the treatment time and the impact of the electron and photon contamination on the delivered dose distribution were studied. The scanned beam was examined both with and without the use of the MLC to delimit the scanned field shape when necessary. All measurements were performed with the MM50 unit at Karolinska University Hospital, which after $20 \mathrm{yr}$ of operation was set to be decommissioned. Unfortunately, this set severe limits on the available beam time and number of possible measurements. Six MM50 units are now operational or under construction in China with many more planned but for logistical and administrative reasons, it was not practical to continue the measurements at these sites.

For cancers that are small and well oxygenated classical uniform beam radiation therapy is generally sufficient. While for large highly hypoxic or radiation resistant tumors, that often reoccur locally, light ions will increasingly be use to maximize the complication free cure. ${ }^{15-17}$ IMRT with scanned beams or other fast, accurate delivery methods alone or in combination with BIOART (Refs. 15, 17) (biologically optimized predictive assay based radiotherapy) approaches could be the treatment of choice for mildly hypoxic tumors of complex local spread. Obviously, the border between these three tumor groups will not be sharp, so fast IMRT will probably be used also for several small tumors to reduce the normal tissue side effects.

In the present study, the first measurements with fast narrow high energy $(50 \mathrm{MV})$ scanned photon beams are presented. Although the measurements were not conducted on a fully developed clinical machine, the results indicate an interesting performance increase. Measurements were made with both photon pencil beam kernels and scanned fields, compared to detailed Monte Carlo (MC) simulations in the case of the photon kernel.

\section{MATERIALS AND METHODS}

\section{II.A. The treatment unit}

All experiments were performed at the Karolinska University Hospital using the racetrack microtron MM50, that in photon mode delivers pulsed electron beams of 5-50 MeV at 50-200 Hz to a bremsstrahlung target placed at the downstream end of the last scanning magnet. Unlike conventional machines where flattening filers are used, the scanning system of the racetrack is designed to allow rectilinear scanning in two perpendicular directions, used to create homogeneous fields of $32 \times 40 \mathrm{~cm}^{2}$ by scanning the incident electron beam on the bremsstrahlung target in a circular conic pattern. This results in a uniform conically divergent photon beam with a diameter close to $50 \mathrm{~cm}$ at isocenter. The desired shape of the radiation field is then limited by the two blocks and 64 double focused collimator leafs. The standard bremsstrahlung target is of full range type made of tungsten and copper so no primary electrons can penetrate through the target. The photon generated leptons (electrons and positrons) produced in the downstream part of the full range target are removed from the beam using a purging magnet placed right after the last scanning magnet and the bremsstrahlung target. A segmented transmission monitor, placed after the purging magnet, is used to verify both the delivered dose and the position of the bremsstrahlung lobe. ${ }^{6}$ For the present experiments, we have instead used a 3-6 mm beryllium transmission target, which only transforms a fraction of the electron energy into bremsstrahlung but produces an intense narrow photon beam with a hard bremsstrahlung spectrum, containing only a small amount of low energy photons. Since the high energy electron beam transmitted through the beryllium target could damage the transmission monitor, it was removed along with the wedge filter selector. Unless otherwise specified the treatment head was filled with helium under normal pressure during these experiments. The interlock safety system of the MM50 was modified in order to run the machine with the new setup. It was, therefore, not possible to run the machine in clinical mode, which normally would have allowed the internal software to regulate the pulse height and length to have a steady dose rate during long irradiation periods.

\section{II.B. Detectors and film}

The film used was of the EDR2 type, which has a low energy dependence and can accept doses up to 6 Gy. ${ }^{18}$ EBT type film would have been an interesting option but at the time the required optical scanner hardware was not available. All films were from the same batch and processed with a Kodak RP X-OMAT film processor following the manufacturers quality routines and then analyzed with a VXR-16 dosimetry pro scanner. The diode detector used was of the P type from Scanditronix, specifically made for photon measurements with the effective measuring point $0.5 \mathrm{~mm}$ into the detector and an active area of $2.5 \times 2.5 \mathrm{~mm}^{2}$. Film dosimetry systems have a high spatial resolution and the dose distributions are less sensitive to variations in the intensity of the intrinsic electron beam during the measurement. $\mathrm{P}$ type diodes have good spatial resolution and are less sensitive to low energy scattered photons than film dosimetry. A cylindrical ion chamber, Rk1, was also used with a Keithley electrometer model 617.

\section{II.C. Spot matrix}

In order to achieve the desired dose distribution using scanned beams, a close Gaussian fit of the photon pencil beam (measured with film at isocenter below $80 \mathrm{~mm}$ of PMMA) was used as input for an inverse planning iterative deconvolution scheme. ${ }^{19}$ The resulting optimal dose distribution minimized the overdosage needed at the edges and corners, to make sure that there is no underdosage in the target volume. This algorithm also minimizes the dose delivery outside of the target volume. The iterative deconvolution scheme delivers a quasicontinuous pencil beam intensity distribution across the entire field, which is not practical from a scanning time point of view. Therefore, the spot matrix was discretized to a small number of equal intensity pencil beams spots using a Levenberg-Marquardt optimizer with an analytical approximation of the pencil beam kernel and the 
associated analytical derivatives of the kernel. For a $10 \times 10$ $\mathrm{cm}^{2}$ field with a $3 \mathrm{~mm}$ Be target, the number of accelerator pulses needed to deliver a homogeneous dose distribution across the entire field was typically around 50. For these experiments, a spot matrix of 100 spots was used to cover a $10 \times 10 \mathrm{~cm}^{2}$ field since it is then less sensitive to errors in the placement and dose of the individual beam spots. This corresponds to two full field scans per second of the treatment time at $200 \mathrm{~Hz}$.

\section{II.D. Experimental setup}

For the diode measurements a RFA300plus system was used with a $97 \times 66 \times 83 \mathrm{~cm}^{3}$ water phantom. The diode system was connected to the scan computer of the MM50 so that each measurement value was recorded after five full scans or 500 pulses. To have a maximum separation of the bremsstrahlung beam and the transmitted electron beam, the electrons were deflected in the opposite direction to the scanning of the photon beam as seen in Fig. 1(a). The block collimator was fully extended and used as an electron collector, covering half the treatment area. The strength of the present purging magnet could not be varied during the irradiation. The part of the Gaussian distributed transmitted electron beam that missed the edge of the block collimator, therefore, varied with the position of the scanned photon beam. When using the thin target technique, the bremsstrahlung generated in the vacuum window consisting of $0.05 \mathrm{~mm}$ beryllium could be detected as an increase in the dose distribution along the isocenter axis. Since the vacuum window is placed before the cross plane scan magnet, every scan position also results in a bremsstrahlung contribution from the vacuum window projected to the isocenter axis. To avoid the influence of the vacuum window bremsstrahlung, both the photon kernel and scanned beams measurements were conducted $8.5 \mathrm{~cm}$ away from the isocenter axis [cf. Fig. 1(a)].

\section{II.D.1. Bremsstrahlung kernel profiles and electron contamination}

Transverse beam profiles of the dose distribution from 3 $\mathrm{mm} \mathrm{Be}, 6 \mathrm{~mm} \mathrm{Be}$, and $\mathrm{WCu}$ bremsstrahlung targets were measured with diodes at the isocenter, $95 \mathrm{~mm}$ below the water surface of the phantom as seen in Figs. 1(a) and 1(b). The photon beam was scanned $120 \mathrm{~mm}$ in the cross direction and the purging magnet operated at maximum strength. Film measurements were taken at isocenter with $80 \mathrm{~mm}$ PMMA in front and behind the film.

\section{II.D.2. Percentage depth dose}

Depth dose distributions were measured along the oblique central axis for both the elementary photon kernel and a uniform scanned dose distribution in a $10 \times 10 \mathrm{~cm}^{2}$ area. Film measurements were taken with the film tightly pressed between $80 \mathrm{~mm}$ PMMA on each side and angled so that the photon kernel/field entered tangentially to the film. The isocenter was placed $80 \mathrm{~mm}$ in the film plane. Diode measurements were made in water, along the oblique central axis of the radiation field, with an $S S D$ of $90.5 \mathrm{~cm}$.

\section{II.D.3. Beam profiles}

The standard radiation field shape during the measurements was $10 \times 10 \mathrm{~cm}^{2}$ with a uniform dose distribution. This field was then used both with or without the MLC delineating the area and also with 1-2 MLC leafs extended into the $10 \times 10 \mathrm{~cm}^{2}$ area. When the MLC was used, some measurements were done with extra spots on the edges of the MLC to sharpen the penumbra. These extra spots were not part of the calculated spot matrix but added on a trial and error basis, since the MLC collimation was not accounted for in the present inversion algorithm. To examine the capability of the scanning system to create complex field profiles without the MLC a cardioid shaped dose distribution was used.

\section{II.E. Monte Carlo simulations}

To compare with the experimental results, Monte Carlo simulations were run taking the magnetic field of the scan and purging magnet into account. The Monte Carlo simulations were made using GEANT4 version 9.2.3 (released 19 December 2009) with G4EmStandardPhysics. This version includes a more accurate multiple scattering model, G4UrbanMscMode193, that has been tuned to fit experimental results of the angular distribution from $13 \mathrm{MeV}$ and $20 \mathrm{MeV}$ electrons scattered in thin foils. ${ }^{20}$ The treatment head geometry used in the simulations is given in Fig. 1(a). The stationary magnetic field from the purging magnet, used during the experiments, was simulated in detail using OPERA3D/TOSCA and was incorporated into the Monte Carlo simulations. This allowed detailed studies of the scattered high energy electrons and the influence of the magnetic field of the purging magnet on the delivered dose distribution. The root-mean-square scattering angle $\sqrt{\bar{\theta}^{2}}\left(z_{0}\right)$ and root-meansquare radial spread $\sqrt{\bar{r}^{2}}\left(z_{0}\right)$ were estimated to be $4.2 \mathrm{mrad}$ and $0.39 \mathrm{~mm}$ when incident on the bremsstrahlung target of the MM50 racetrack at Karolinska ${ }^{11}$ and were used as initial values. A total of $10^{9}$ histories were simulated resulting in statistical uncertainty of less than $0.5 \%$ at maximum dose. Monte Carlo simulations were also done to examine the source of the stray radiation in the radiation field.

\section{RESULTS}

\section{III.A. Bremsstrahlung kernel profiles and high energy electron contamination}

The bremsstrahlung kernel profiles measured at isocenter with diodes below $95 \mathrm{~mm}$ of water from bremsstrahlung targets of $3 \mathrm{~mm}, 6 \mathrm{~mm}$ beryllium, and $5 \mathrm{~mm}+7.5 \mathrm{~mm}$ tungsten + copper (full range target) normalized to the peak photon yield ${ }^{10}$ are shown in Fig. 1(b). The full width at halfmaximum (FWHM) of the $3 \mathrm{~mm}$ beryllium target was $34 \mathrm{~mm}$ at isocenter, which is very close to previous simulations ${ }^{10}$ of $33 \mathrm{~mm}$ at an SSD of $100 \mathrm{~cm}$. Interestingly, the peak forward bremsstrahlung intensity is higher at $6 \mathrm{~mm} \mathrm{Be}$ than when using a full range target, due to lower photon attenuation in the target. An elevated dose region is seen on the left side of the peak for the 3 and $6 \mathrm{~mm} \mathrm{Be}$ kernels. This is the direction where the transmitted electrons are deflected, indicating that 
it is caused by the tail of the scattered primary electrons. In Fig. 2, diode and ion chamber measurements of the photon kernel from a $3 \mathrm{~mm}$ Be target at isocenter below $95 \mathrm{~mm}$ water are compared to GEANT4 simulations. The elevated region in the Monte Carlo simulation, as shown in Fig. 2(a), is at around $2.0 \%$, while the diode measurements gave a value of $2.6 \%$ compared to the peak forward photon dose. In the ion chamber measurements, the elevated region was at $2.4 \%$ with a relative standard deviation of $1.8 \%$. Also seen in Fig. 2(a) is a Monte Carlo simulation of the dose distribution from unscattered bremsstrahlung photons, incident on the water phantom, that have not interacted with the block or the MLC. The dose distribution from photons or electrons that have interacted with either the block or MLC is presented in Fig. 2(b). This can be particles that have passed the block or the MLC or been scattered on their edges, and the dose distribution is rather uniform inside the collimated area. Also in Fig. 2(b) is the electron dose distribution deposited in the water phantom by electrons that have not interacted with the block or MLC. This clearly shows that part of the Gaussian distributed electron beam transmitted through the target is insufficiently deflected and directly incident on the water phantom. As shown in Fig. 2(b), the maximum contribution from these electrons is $2.2 \%$ of the peak forward photon dose and it is, in combination with the tail of the photon dose distribution, the main components of the elevated dose region shown to the left in Fig. 2(a). This indicates that the high energy scattered electron component of the dose distribution has been identified by both Monte Carlo and experimentally, since the measured depth dose has the characteristics of just under $50 \mathrm{MeV}$ electrons. The fraction of a percent difference between the diode and Monte Carlo calculations may be due to scatter in the opposite block and other components not considered in the MC simulations. The isodose distribution of the whole pencil beam kernel measured with film is shown in Fig. 3(a).

\section{III.B. Spot matrix}

For a $10 \times 10 \mathrm{~cm}^{2}$ uniform field, the optimal spot density distribution calculated by the deconvolution algorithm was discretized down to 100 spots [see Fig. 3(b)]. The number of spots could be further reduced down to about 50 spots without much loss in accuracy, but for the present experiments it was deemed unnecessary. In Fig. 3(c), the analytical fit of the bremsstrahlung kernel used in the deconvolution algorithm is shown along with the bremsstrahlung kernel measured with film. In order to have a bremsstrahlung kernel without the elevated electron dose region, the measurement was made transverse to the bending plane. Also in Fig. 3(c) is the resulting calculated dose distribution for a $10 \times 10 \mathrm{~cm}^{2}$ uniform field is shown along with the measured values. In the $10 \times 10$ $\mathrm{cm}^{2}$ scanned area, the fit is better than $2 \%$. On the left side in Fig. 3(c), the measured values are considerably below the analytical values since it is blocked by the primary collimator.

\section{III.C. Percentage depth dose}

The measured central axis depth dose distribution from a $3 \mathrm{~mm}$ Be transmission target using diodes is compared with
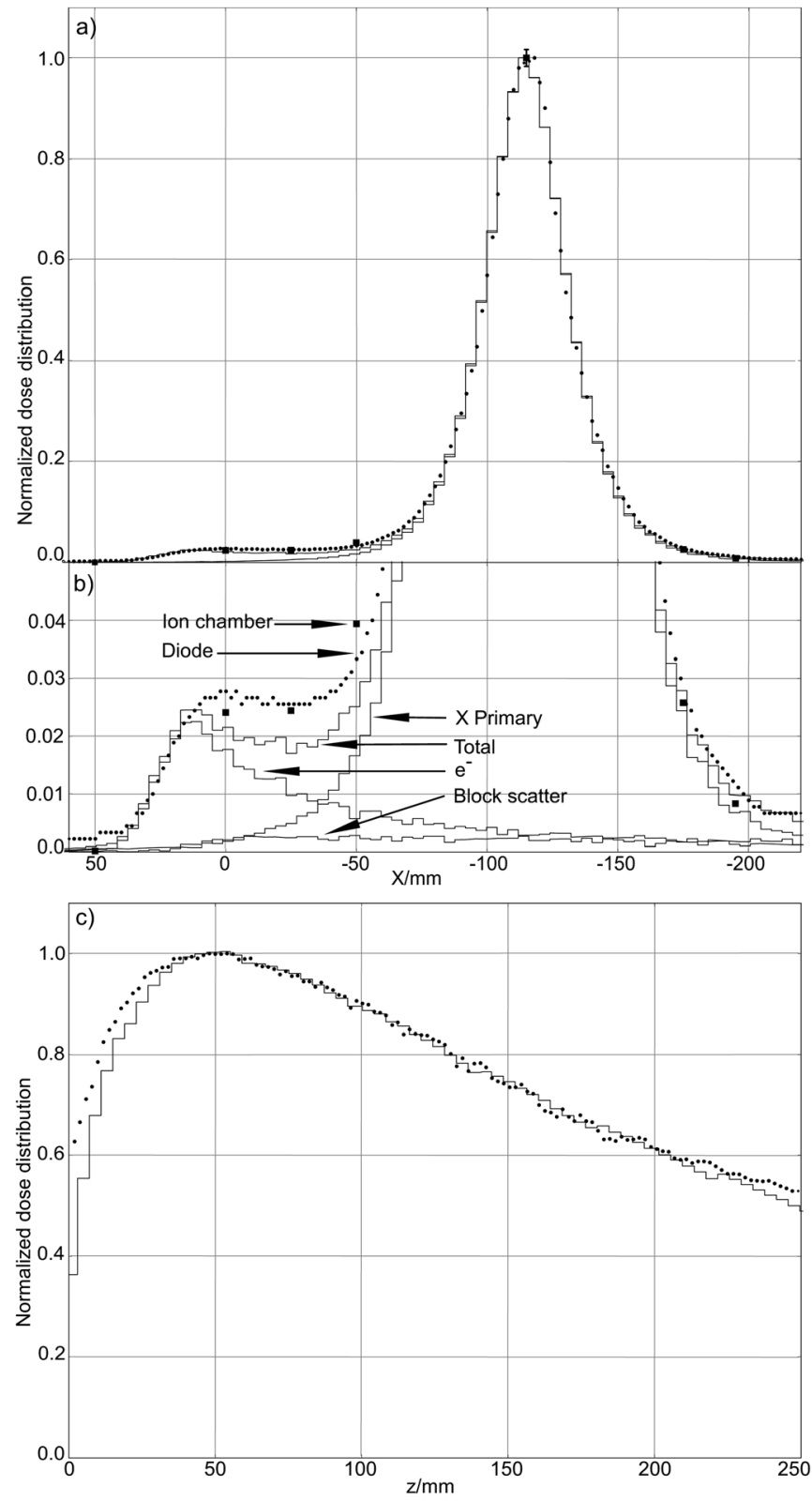

FIG. 2. (a) Diode measurement at isocenter at SSD of $90.5 \mathrm{~cm}$ below $95 \mathrm{~mm}$ of water (dots) and ionization chamber measurements at the same depth (squares). Compared to Monte Carlo simulation of the total dose distribution and of the dose distribution from particles that were photons incident on the water phantom and which did not interact with the block or MLC. (b) The lower region of (a) complemented with two Monte Carlo simulations: dose distribution, normalized to the total dose distribution, from particles that have interacted with the block or MLC. Dose distribution normalized to the total dose distribution, from particles that were electrons incident on the top of the water phantom and had not interacted with the block or MLC. (c) Central axis depth dose distribution of the photon kernel from a $3 \mathrm{~mm} \mathrm{Be}$ bremsstrahlung target obtained with diode in water (dots), and by Monte Carlo simulation.

Monte Carlo simulations in Fig. 2(c). The depth of dose maximum is at $5.5 \mathrm{~cm}$ (SSD $90 \mathrm{~cm}$ ) both for the measurement and the simulation, and the agreement in the deep part of the depth dose distribution is satisfactory. In the build up region, the simulation and the measurement differ somewhat. It seems that the diode measured experimental entrance dose is higher than the simulation results 

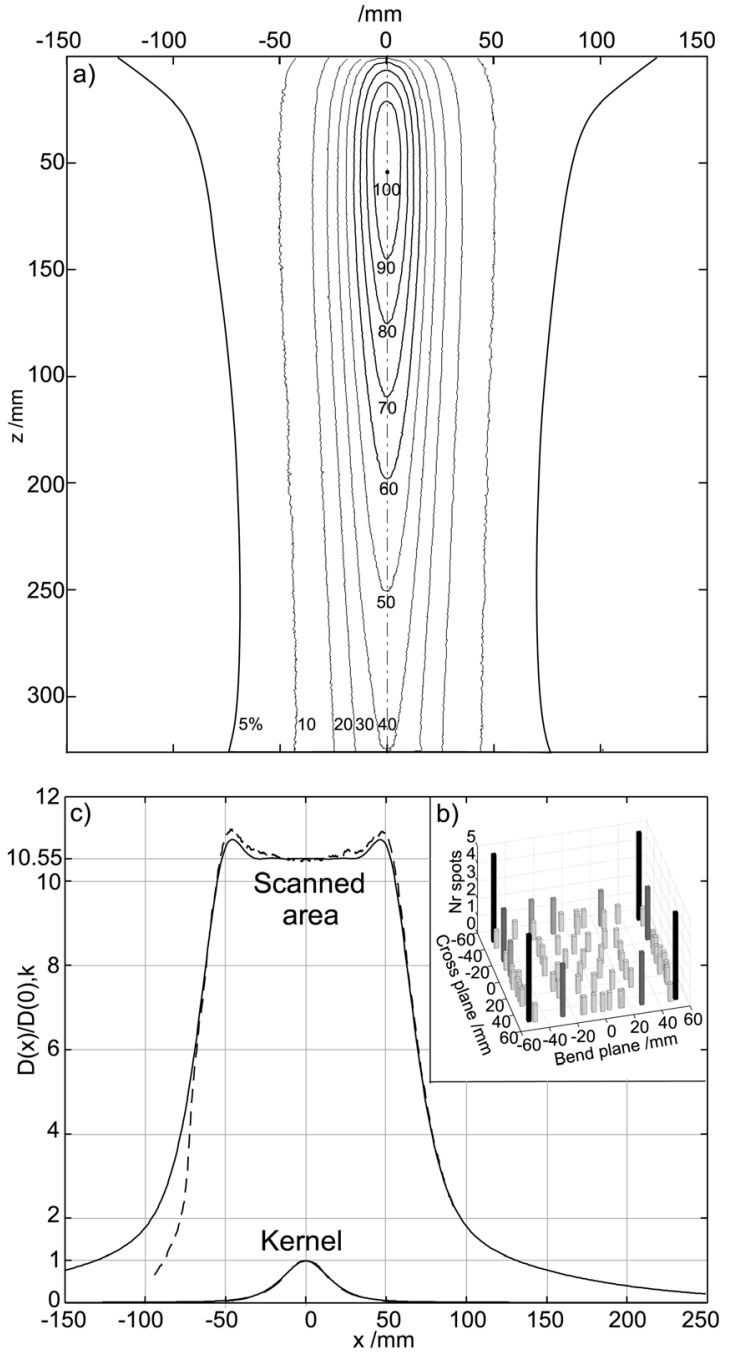

FIG. 3. (a) Isodose diagram for a $50 \mathrm{MeV}$ pencil beam of perpendicular incidence at SSD $90.5 \mathrm{~cm}$ recorded by film corrected for the influence of electron contamination. The pencil beam FWHM in air is $30 \mathrm{~mm}$ and at $10 \mathrm{~cm}$ depth in water $34 \mathrm{~mm}$, both at isocenter. The sharp penumbra and absence of wide tails, characteristic of thick target bremsstrahlung beams, is due to the harder photon energy spectrum and less multiple electron scatter in the target prior to bremsstrahlung production at lower energies. (b) The spot matrix used to create a uniform $10 \times 10 \mathrm{~cm}^{2}$ area; (c) In the lower part, the dashed line shows the measured lateral dose distribution of the photon kernel, while the solid line is the analytical fit to measured bremsstrahlung kernel used by the deconvolution algorithm. In the top part, the solid line is the analytical fit of the kernel convolved with the spot matrix, shown in (b). The dashed line shows the measured dose distribution across the scanned $10 \times 10 \mathrm{~cm}^{2}$ area.

[cf. Fig. 2(c)]. This is strange since we already demonstrated that the measured high energy electron contamination was only around 2\% (see Sec. III A). Possible experimental errors are a droplet of water on top of the detector or a nonlinearity issue and energy dependence of the silicon diode. It could also be due to a low energy electron or photon contamination from components in the treatment head that were not included in the Monte Carlo simulation. An isodose image of a $10 \times 10 \mathrm{~cm}^{2}$ scanned homogeneous beam from a $3 \mathrm{~mm}$ Be target measured with film is shown in Fig. 4(a). The recorded relative entrance dose is fairly high indicating a possible misalignment of the film to the edge of the PMMA
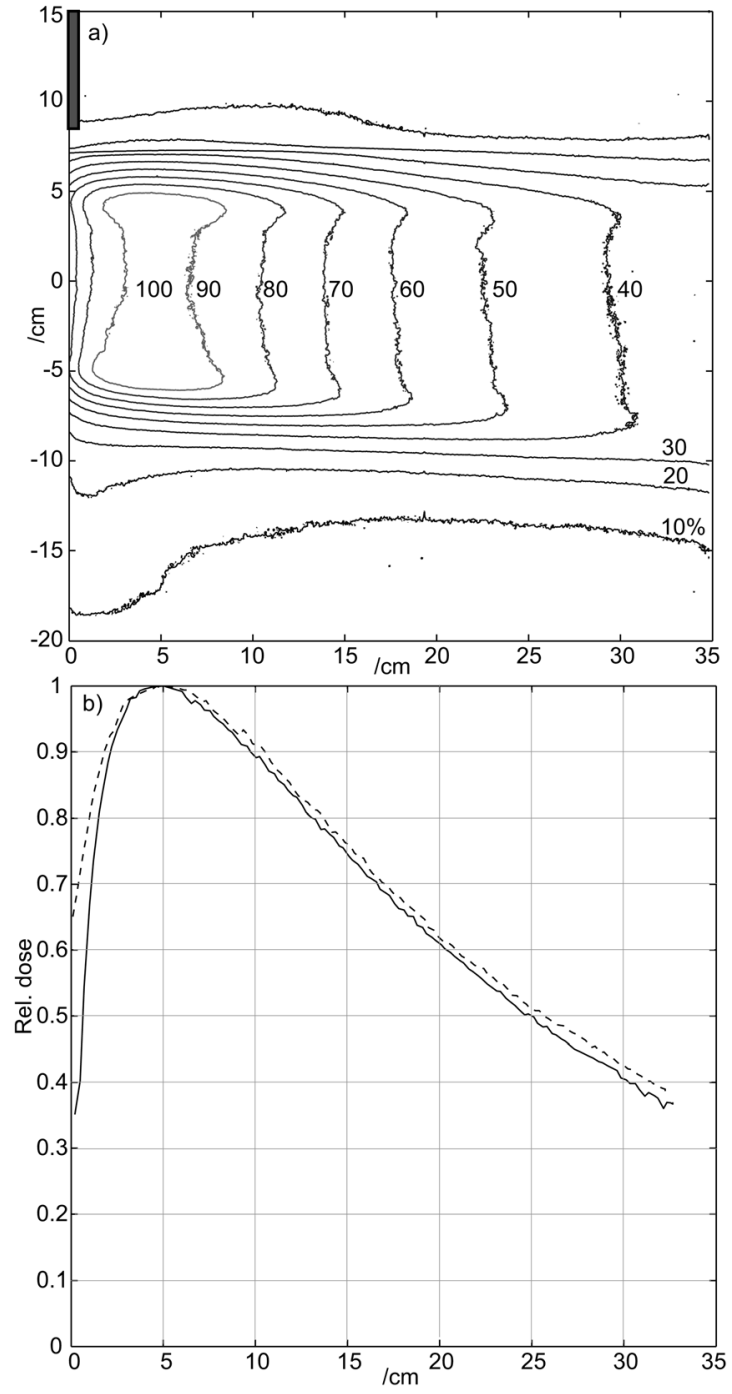

FIG. 4. (a) Isodose distribution of a homogeneous $10 \times 10 \mathrm{~cm}^{2}$ scanned field with a $3 \mathrm{~mm}$ Be target measured with film. The gray area represents the projection of the MLC/Block collimator acting as an electron stopper. (b) Central axis depth dose curves measured with a diode in water for a collimated scanned photon beam $\left(10 \times 10 \mathrm{~cm}^{2}\right.$ at isocenter) from a $3 \mathrm{~mm}$ bremsstrahlung target (dashed line) and a collimated beam $\left(10 \times 10 \mathrm{~cm}^{2}\right.$ at isocenter) from a full range $\mathrm{WCu}$ target (solid line).

slabs. In Fig. 4(b), two diode measurements are shown for a scanned $10 \times 10 \mathrm{~cm}^{2}$ beam with a $3 \mathrm{~mm} \mathrm{Be}$ target (dashed line) and a collimated $10 \times 10 \mathrm{~cm}^{2}$ field using a full range target of $\mathrm{WCu}$ (solid line). The entrance dose with the MM50 using a full range target of $\mathrm{WCu}$ has been carefully measured with extrapolation chamber to about $15 \%$, and the diode measurement presented here for the full range target is in good agreement with diode measurements both by the manufacturer and the hospital. The bremsstrahlung generated in the crude electron collector (the block collimator) will influence the dose distribution more with a scanned beam than with a stationary pencil beam since only a few pulses deliver high photon dose to any point, while all pulses contribute to the scatter and photon leakage from the electron collector. The lower energy bremsstrahlung generated in the electron collector is more isotropic and will thus add up about tenfold in a scanned beam [compare Fig. 3(c)]. The 
above mentioned experimental errors may also be involved here. Unfortunately, due to the limited measurement possibilities before decommissioning the treatment unit these effects could not be studied further.

\section{III.D. Scanned beams}

In Fig. 5, dose distributions using the scanning beam technique, with a $3 \mathrm{~mm}$ Be target, are presented with and without MLC collimation, measured with film at isocenter below 8 $\mathrm{cm}$ of PMMA. Figure 5(a) is an uncollimated scanned $10 \times 10 \mathrm{~cm}^{2}$ beam, derived using the minimum overdosage inverse planning algorithm, with slight overdosage near the edges and corners clearly seen. The same field but collimated along the edges is shown in Fig. 5(b), creating the typical rounded edge effect due to out scatter of electrons and photons. The flatness measured along the major axes was within $3 \%$ for the collimated case, which is comparable to or better than the flatness achieved with conventional systems using flattening filters. ${ }^{21}$ By adding extra pencil beam spots along the edges as shown in Fig. 5(c), the delivered dose profile can be markedly sharper at the edges of the field and thus even better produce the desired homogeneous dose distribution. Here, the added spots created soft peaks along the edges, which makes the flatness go up to about $4 \%$. In Fig. $5(d)$, the relative dose profiles are compared for the different cases. Although the extra spots overcompensated the loss of scattered radiation from the collimated area, the created peaks show that this is also an efficient way to compensate for uncertainties in beam tumor alignment that are always present. $^{22-24}$

In Figs. 6(a)-6(c), film measurements are presented for different techniques of creating a uniform dose distribution in a $10 \times 10 \mathrm{~cm}^{2}$ field with one leaf extended into the field. In Fig. 6(d), diode measurements over a collimated $10 \times 10$ $\mathrm{cm}^{2}$ area with two extended MLC leaves are compared, using a full range target with a circular scan pattern and a 3 $\mathrm{mm}$ Be target with the scan pattern shown in Fig. 3(b). A scanned field, built up by $3 \mathrm{~mm}$ Be photon kernels, with collimation has a penumbra of $\left(\mathrm{a}, P_{20,80}=9 \mathrm{~mm}\right)$ and with added extra spots on the edges of the MLC (b, $P_{20,80}$ $=9 \mathrm{~mm}$ ). When using a full range target, the penumbra is (c, $P_{20,80}=10 \mathrm{~mm}$ ), while a scanned field, built up by $3 \mathrm{~mm}$ Be photon kernels, without collimation gives a penumbra of $P_{20,80}=36 \mathrm{~mm}$. The penumbra using the scanned beam technique is very similar to the one using a full range target with collimation but the target area displays a much flatter dose distribution. The relative dose outside the target area is higher with the scanned beam technique especially when extra spots are added on the edges [cf. Fig. 6(d)]. Indicating the presence of scattered radiation from the imperfect hardware used here since, at least far outside the field edge, the collimator leakage with scanned beams should be reduced because no primary photons are directed there.

To illustrate both the capabilities and limitations of using only the scanned photon beam to modulate the dose distribution, without the use of the MLC, a cardioid shaped dose distribution was used. Since it is not generally possible to achieve the resultant very sharp dose gradients with conventional dose delivery methods, it was selected in order to test the limits of using narrow scanned photon beams to shape arbitrary dose distributions. In Fig. 7, the achievable dose distribution using the minimum overdosage inverse planning algorithm is shown along with the measured dose distribution. As can be seen the scanned beam gives the possibility to shape the resulting dose distribution in the target volume with the limitations set by the present rather broad photon pencil beam kernel. The dose outside the target volume is too high in this case since no collimation was used, which indicates that although most of the dose distribution can be shaped with the scanned beam alone, an MLC or a PMLC is useful as a penumbra trimmer to sharpen the target volume. In Fig. 7(c), the isodose curves from the analytically planned and measured dose distribution are shown. The markedly straight appearance of the outer isodose lines for the measured distribution on the bottom and left side is due to the proximity of the edge of the treatment head collimation. In the center, the analytical and measured curves fit within a few percent, while at the right side, the measured values exceed the analytical values. This effect may be partly attributed to the uncompensated bremsstrahlung from the vacuum window, which lies along the isocenter axis located at around $8.5 \mathrm{~cm}$ in Fig. 7(c). Also for these experiments, the same analytical kernel profile was used when calculating the spot matrix, while the real kernel profiles changes over the target area due to the geometrical setup. ${ }^{11}$

\section{III.E. IMRT treatment time}

The treatment time, when using a thin target, is dependent on the pulse repetition frequency, beam intensity, pulse length, the integral yield of the bremsstrahlung target, and the number of spots in the scan matrix. Although the peak forward yield of a $3 \mathrm{~mm}$ Be target is lower (83\%) than the peak forward yield of a full range target, consisting of $5 \mathrm{~mm}$ $\mathrm{W}$ and $7.25 \mathrm{~mm} \mathrm{Cu}$, the main reason for the increase in treatment time lies in the much lower integral yield and the larger number of beam spots needed to cover the whole target volume. For a homogeneous dose distribution in a $10 \times 10 \mathrm{~cm}^{2}$ area the increase in treatment time is about 3.6 times compared with the standard scanning pattern with a full range target. For the scanned dose distributions presented here, homogeneous $10 \times 10 \mathrm{~cm}^{2}$ and a cardioid shape, the time needed to build up the field (one scan) was $0.5-1.36 \mathrm{~s}$ with 100 and 272 spots, respectively. The treatment time is then mainly depending on the intrinsic electron beam intensity and since it is quite low in this case (less than $1 \mu \mathrm{A}$ ), and the treatment machine was designed to deliver sufficiently high dose rate, even for photon beams of very low energy, the treatment time can easily be normalized to the full range target case. ${ }^{10}$

\section{DISCUSSION}

The present purging magnet was not designed to deflect the primary electrons with energies up to $50 \mathrm{MeV}$, and the experimental setup was, therefore, limited by the deflection 

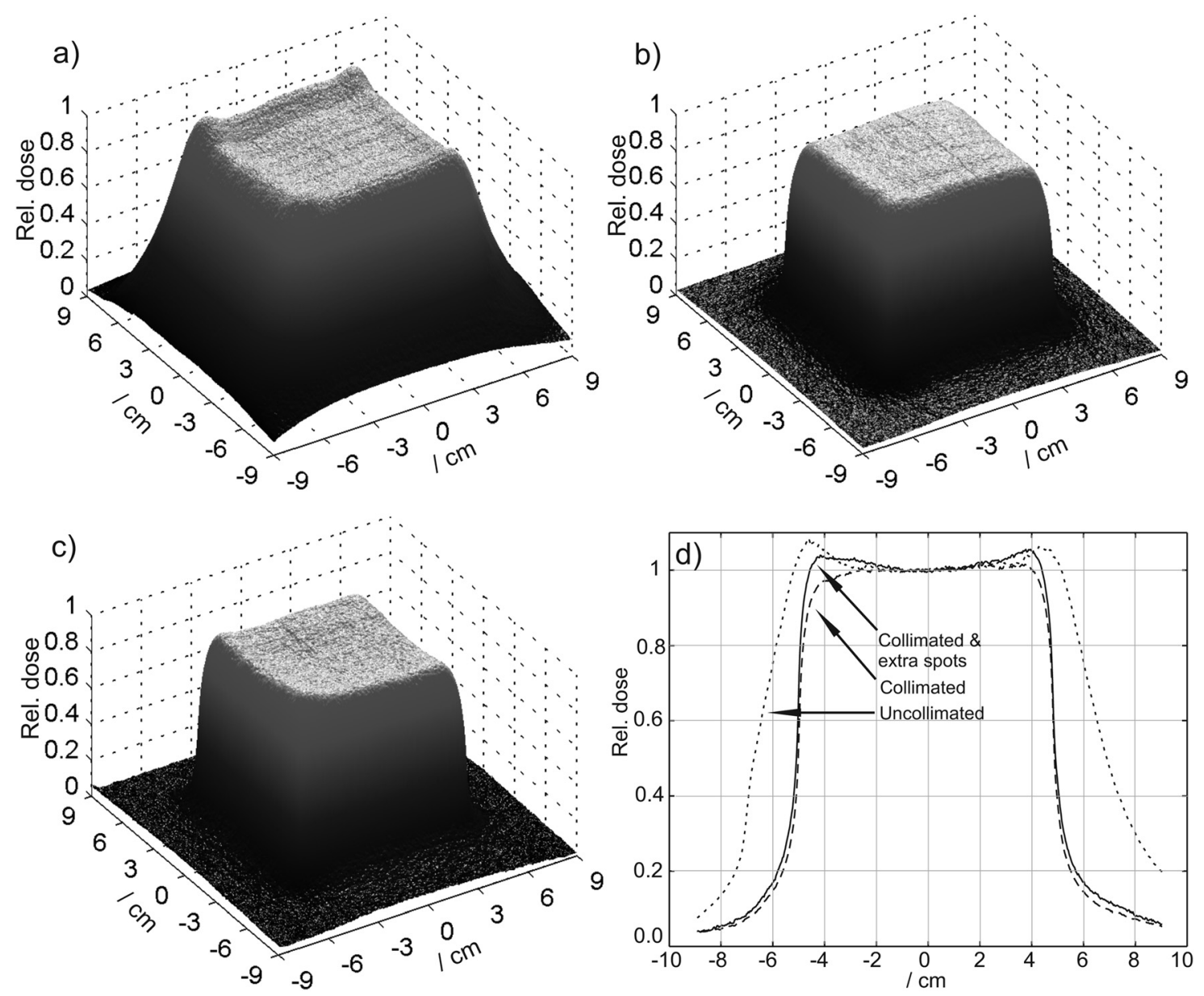

FIG. 5. Dose distributions in an $18 \times 18 \mathrm{~cm}^{2}$ area measured with film at isocenter below $8 \mathrm{~cm}$ of PMMA. Scanned 3 mm Be photon kernels were used to build up a uniform dose distribution within a $10 \times 10 \mathrm{~cm}^{2}$ area. (a) Uncollimated; (b) collimated; (c) collimated with extra spots on the edges of the MLC; (d) dose distribution across the central part of the $10 \times 10 \mathrm{~cm}^{2}$ area for the three cases.

capabilities of this magnet. Since the strength of the purging magnet could not be varied during an irradiation, the deflection of the transmitted electrons varied with the scanning of the photon beam. With scanned beams, the delivered dose distribution is built up by a large number of spot positions, but most photon spots contribute only marginally to the delivered dose at any position. Bremsstrahlung and electron contamination from treatment head components are always present, and it is important to minimize it. By altering the strength of the purging magnet the transmitted electron beam can be deflected to the same spot on a dedicated electron collector in the upper part of the treatment head regardless of the scan position of the photon beam. This would reduce the electron and bremsstrahlung contamination, which was still rather low during the present measurements. A scanning and purging magnet system capable of deflecting the transmitted electron beam when using the thin target technique has been developed for energies 50-75 $\mathrm{MeV}{ }^{9,25}$ With an intrinsic electron energy of $75 \mathrm{MeV}$ and an SID of $75 \mathrm{~cm}$, the FWHM is down to about $10-11 \mathrm{~mm}$, which corresponds approximately to the geometric resolution of $5 \mathrm{~mm}$ wide collimator leafs and thus further improve dose delivery modulation.

Even if the present measurements are not made on a fully developed clinical machine, the measured beam spot of 34 mm FWHM is quite useful for many IMRT applications as shown in Figs. 4, 6, and 7. The present measurements thus show that fast IMRT is possible with narrow scanned photon beams as longs as the transmitted primary electron beam can be sufficiently deflected and trapped. When only the MLC is used the dose distribution has rounded corners due to the lack of scatter equilibrium in the collimated area, a common problem of most clinical photon dose distributions. We have here examined the possibility to deliver extra beam spots along the edges of the MLC to compensate for this loss, and thereby, getting much sharper dose distributions near the field edges. Scanned IMRT with penumbra trimming multileaf collimation should, therefore, be a very interesting possibility in the future development of biologically optimized IMRT. ${ }^{26}$ In this study, the focus was on beam scanning and tumor dose delivery. Since the Monte Carlo calculations agreed well with the measured beam profile including the elevated dose region, most likely due to high energy electron contamination on the electron deflection side, it can be used to estimate the mean relative dose between $0-4 \mathrm{~mm}$ of depth to about 35\% for the scanned photon kernel [cf. Fig. 2(c)]. The origin of the measured higher relative entrance dose of the photon kernel is most likely in the interaction of the electron beam transmitted through the target with components of 

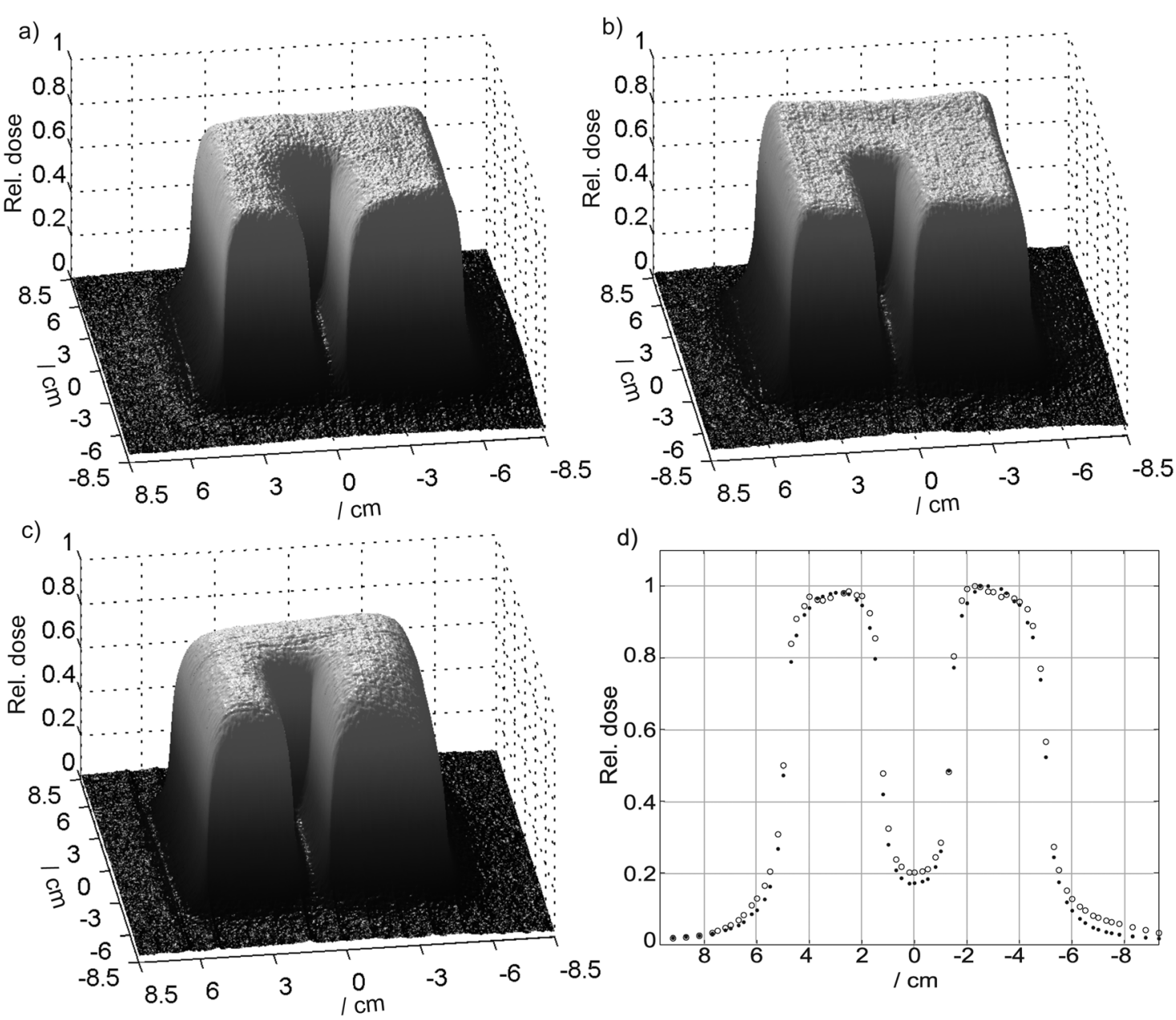

d)

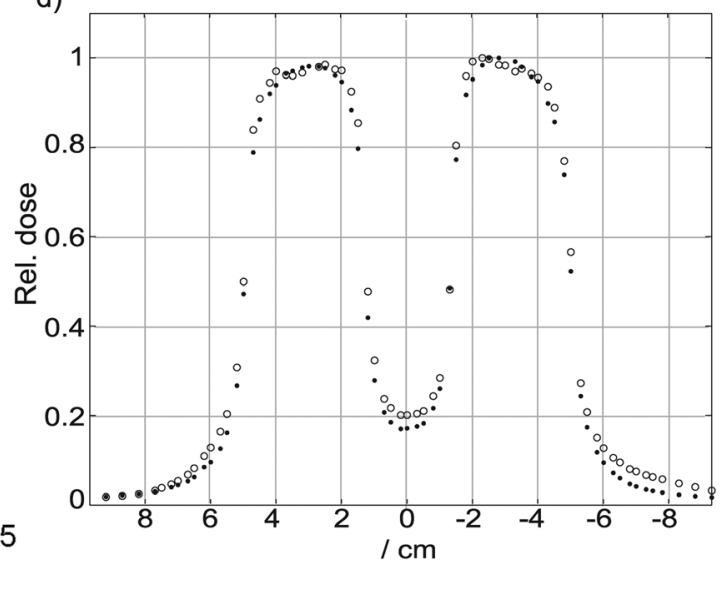

FIG. 6. Dose distributions in a $17 \times 17 \mathrm{~cm}^{2}$ area measured with film at isocenter below $8 \mathrm{~cm}$ of PMMA. In (a), (b), (c) bremsstrahlung targets of 3 mm Be and a full range target was used to build up a homogeneous dose distribution inside a collimated $10 \times 10 \mathrm{~cm}^{2}$ area with one leaf extended into the field. (a) 3 mm Be as target with no spots on the edge of the MLC; (b) $3 \mathrm{~mm} \mathrm{Be}$ as target with extra spots added on the edges of the MLC; (c) full range target and a circular scan pattern; (d) diode measurements at isocenter, below $95 \mathrm{~mm}$ of water, across the $10 \times 10 \mathrm{~cm}^{2}$ collimated area with two extended MLC leaves. Scanned 3 $\mathrm{mm}$ Be bremsstrahlung beams with extra spots on the edges of the MLC (circles) compared with a full range target using a circular scan pattern (dots).

the treatment head. In principle, narrow photon pencil beam scanning IMRT reduces the leakage always obtained with filtered beam IMRT. This is due to the fact that the photon fluence incident on the collimators is minimized since the narrow scanned photon beam is practically always directed on the target volume where the collimator is fully open and, therefore, very little photon fluence needs to be blocked by the collimator.

The recently introduced and quite popular single arc rotation therapy technique is by necessity suboptimal with regard to its intensity modulation, ${ }^{27}$ since open weakly modulated beams are generally used. The fast scanned beam IMRT treatment technique discussed here can rapidly modulate the dose delivery inside the dynamic collimator opening improving the dose delivery for complex target volumes when using rapid arc delivery. As such it is potentially well suited to improve single arc rotation therapy.

The bremsstrahlung from a thin target has a higher mean energy and thus will give a deeper dose maximum and depth dose distribution than a full range target. This is not only of interest for treatment planning but also for the level of induced positron activity in the body, which can be used for in vivo treatment planning and treatment verification using PET-CT imaging. ${ }^{17,28,29}$ Due to a peak photonuclear cross section around $24 \mathrm{MeV}$ (for carbon and oxygen) an intrinsic electron energy of $75 \mathrm{MeV}$ would be ideal for PET-CT imaging since the effective photon energy is about $1 / 3$ of the energy of the primary electrons with the additional advantage of a FWHM of the photon pencil beam of about $10 \mathrm{~mm}$ further improving the dose delivery modulation. This has the additional advantage that the narrow scanned pencil beam will be as small as around $10 \mathrm{~mm}$ and thus allow further improve dose delivery modulation. Also, when the narrow photon pencil beam is scanned, the average photon energy fluence is much more homogeneous than when using a broad beam generated by a thick target and flattening filters. ${ }^{30}$ In fact, a narrow scanned high energy photon beam is the ideal activation source since the activation efficiency and the effective photon energy will be more uniform all over the target volume due to the absence of flattening filters and low multiple electron scattering in the thin beryllium target. $^{31}$ This makes narrow high energy beam scanning very 

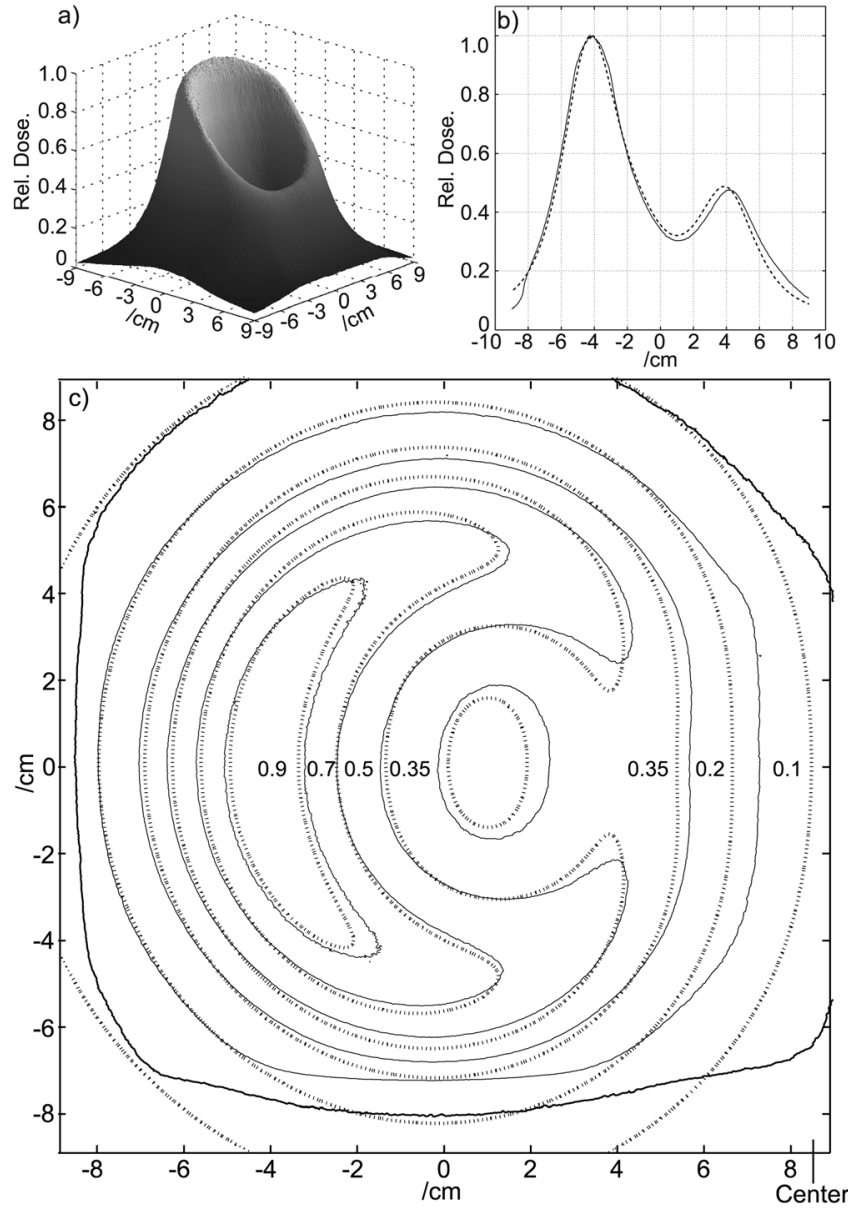

FIG. 7. (a) Measured dose distribution with film at isocenter below $80 \mathrm{~mm}$ of PMMA; (b) achievable dose distribution, along a line, for an oblique cardioid shape from the inverse planning software (dashed) and the resulting measured dose distribution (solid). (c) Isodose curves for the analytical calculated cardioid shape and the measured distribution (solid). The center of the available treatment area is marked, which is where the impact of the beryllium vacuum window can be seen.

interesting not only from a physical, dosimetric, and technical point of view but also for PET-CT treatment verification and biologically adaptive therapy optimization. ${ }^{16,17,26,28,29}$

\section{ACKNOWLEDGMENTS}

Support from the 6th framework program BIOCARE and the Cancer society of Stockholm are gratefully acknowledged. The authors are also grateful to the Karolinska Hospital for their support and the use of their facilities during this work and for the technical assistance from Scanditronix Wellhöfer AB.

\footnotetext{
${ }^{\text {a) }}$ Author to whom correspondence should be addressed. Electronic mail: bjorn.andreassen@ki.se

${ }^{1}$ P. Källman, B. Lind, A. Eklöf, and A. Brahme, "Shaping of arbitary distributions by dynamic multileaf collimation," Phys. Med. Biol. 11, 12911300 (1988)

${ }^{2} \mathrm{~A}$. Brahme, "Optimization of stationary and moving beam radiotherapy techniques," Radiother. Oncol. 12, 129-140 (1988).

${ }^{3}$ T. Bortfeld, "IMRT: A review and a preview," Phys. Med. Biol. 51, R363-R379 (2006).
}

${ }^{4}$ R. Svensson, S. Larsson, I. Gudowska, R. Holmberg, and A. Brahme, "Design of a fast multileaf collimator for radiobiological optimized IMRT with scanned beams of photons, electrons, and light ions," Med. Phys. 34, 877-888 (2007).

${ }^{5}$ A. Brahme, I. Gudowska, S. Larsson, B. Andreassen, R. Holmberg, and R. Svensson, "Application of Geant4 in the development of new radiation therapy treatment methods," in Proceedings of the 9th Conference on Astroparticle, Particle and Space Physics, Detectors and Medical Physics Applications (World Scientific Publishing Co. Pte., Singapore, 2006), pp. 451-461.

${ }^{6} \mathrm{~A}$. Brahme, "Design principles and clinical possibilities with a new generation of radiation therapy equipment," Acta Oncol. 26, 403-412 (1987).

${ }^{7} \mathrm{~A}$. Brahme, "Biological and physical dose optimization in radiation therapy," Accomplishment in Cancer Research (New York, 1992), pp. 265-298.

${ }^{8}$ M. Blomquivist, A. Sätherberg, M. Karlsson, and B. Zackrisson, "Scanned intensity modulation for 50 MV photons," Phys. Med. Biol. 43, 11851197 (1998).

${ }^{9}$ R. Svensson, B. K. Lind, and A. Brahme, "Beam characteristics and clinical possibilities of a new compact treatment unit design combining narrow pencil beam scanning segmental multileaf collimation," Med. Phys. 25, 2358-2369 (1998).

${ }^{10}$ R. Svensson, M. Åsell, P. Näfstadius, and A. Brahme, "Target, purging magnet and electron collector design for scanned high energy photon beams," Phys. Med. Biol. 43, 1091-1112 (1998).

${ }^{11}$ R. Svensson and A. Brahme, "Effective source size, yield and beam profile from multi-layered bremsstrahlung targets," Phys. Med. Biol. 41, 1353 1379 (1996).

${ }^{12} \mathrm{R}$. Rosenqvist, "Application of inversion methods in the implementation of scanned photon beams for radiation therapy," M.Sc. thesis, Royal Institute of Technology and Karolinska Institute, 1990.

${ }^{13}$ B. K. Lind and P. Källman, "Experimental verification of an algorithm for inverse radiation therapy planning," Radiother. Oncol. 17, 359-368 (1990).

${ }^{14} \mathrm{R}$. Sjögren and M. Karlsson, "Electron contamination in clinical high energy photon beams," Med. Phys. 23, 1873-1881 (1996).

${ }^{15}$ A. Brahme, "Development of radiobiologically based therapy optimization and target definition," in Proceedings of the Fourth International Symposium on the Lymphatic System - New Developments in Oncology and IMRT, edited by I. C. Kiricuta Limburg, Germany, 2004 pp. 155-175.

${ }^{16} \mathrm{~A}$. Brahme, "Fractionation and biologically optimized IMRT using in vivo predictiv assay based radiation therapy (BioArt)," in Proceedings of the Fifth International Symposium on the Lymphatic System, edited by I. C. Kiricuta (Limburg, Germany, 2005), pp. 1-36.

${ }^{17} \mathrm{~A}$. Brahme, "Potential developments of light ion therapy: The ultimate conformal treatment modality," Rad. Sci. 52, 8-31 (2009).

${ }^{18} \mathrm{~A}$. J. Olch, "Dosimetric performance of an enhanced dose range radiographic film for intensity-modulated radiation therapy quality assurance," Med. Phys. 29, 2159-2168 (2002).

${ }^{19} \mathrm{~B}$. K. Lind, "Properties of an algorithm for solving the inverse problem in radiation therapy," Inverse Probl. 6, 415-426 (1990).

${ }^{20}$ B. Faddegon, I. Kawrakow, Y. Kubyshin, J. Perl, J. Sempau, and L. Urban, "The accuracy of EGSnrc, GEANT4 and PENELOPE Monte Carlo systems for the simulation of electron scatter in external beam radiotherapy," Phys. Med. Biol. 54, 6151-6163 (2009).

${ }^{21}$ J. Olofsson, T. Nyholm, A. Ahnesjö, and M. Karlsson, "Optimization of photon beam flatness for radiation therapy," Phys. Med. Biol. 52, 17351746 (2007)

${ }^{22}$ K. Lind, P. Källman, B. Sundelin, and A. Brahme, "Optimal radiation beam profiles considering uncertainties in beam patient alignment," Acta Oncol. 32, 331-342 (1993).

${ }^{23}$ L. Löf, B. Lind, and A. Brahme, "Optimal radiation beam profiles considering the stochastic process of patient positioning in fractionated radiation therapy," Inverse Probl. 11, 1189-1209 (1995).

${ }^{24} \mathrm{~J}$. Unkelbach and U. Oelfke, "Inclusion of organ movements in IMRT treatment planning via inverse planning based on probability distributions," Phys. Med. Biol. 49, 4005-4029 (2004).

${ }^{25}$ B. Andreassen, R. Svensson, R. Holmberg, H. Danared, and A. Brahme, "Development of an efficient scanning and purging magnet system for IMRT with high energy photon beams," Nucl. Instrum. Methods Phys. Res. A 612, 201-208 (2009).

${ }^{26} \mathrm{~A}$. Brahme, "Biological modelling for 3-dimensional in vivo predictive assay based adaptive radiation therapy (BIOART)," Radiother. Oncol. 73 (Suppl.1) s62 (2004) 
${ }^{27}$ T. Bortfeld and S. Webb, "Single-arc IMRT," Phys. Med. Biol. 54, N9N20 (2008).

${ }^{28}$ S. Janek, R. Svensson, J. Jonsson, and A. Brahme, "Development of dose delivery verification by PET imaging of photonuclear reactions following high energy photon therapy," Phys. Med. Biol. 51, 5769-5783 (2006).

${ }^{29} \mathrm{~A}$. Brahme, "Biologically optimized 3-dimensional in vivo predictive assay based radiation therapy using positron emission tomography- computerized tomography imaging," Acta Oncol. 42, 123-136 (2003)

${ }^{30}$ A. Sätherberg and M. Karlsson, "Calculation of photon energy and dose distribution in a $50 \mathrm{MV}$ scanned photon beam for different target configurations and scan patterns," Med. Phys. 25, 236-240 (1998).

${ }^{31}$ M. Karlssson, H. Nyström, and H. Svensson, "Photon beam characteristics on the MM50 racetrack mictrotron and a new approach for beam quality determination," Med. Phys. 20, 143-149 (1993). 